\title{
DSM-5 and Clinical Social Work: Mental Disorder and Psychological Justice as Goals of Clinical Intervention
}

\author{
Jerome C. Wakefield
}

Published online: 21 May 2013

(c) Springer Science+Business Media New York 2013

This issue of Clinical Social Work Journal explores facets of the new DSM-5 and the broader relationship between psychiatric diagnosis and clinical social work. The contributors, scholars from both within and outside social work, offer diverse and provocative insights and perspectives. Before introducing the papers, I briefly suggest some overarching issues facing clinical social work in confronting the new DSM.

\section{Medicalization, Brain-Disease Ideology, and Diagnostic Expansiveness}

Currently, the following three processes are happening all at once in psychiatric diagnosis. First, to ensure that psychiatry remains part of medicine, there is a continued insistence that psychiatric disorder is disorder in the same sense as medical disorder. Classifying a condition as a mental disorder thus implies that something has gone wrong with some psychological process or mechanism that is not functioning in the way it is biologically designed to function (i.e., there is an internal dysfunction). This is built right into the definition of mental disorder in the DSM's introduction. Second, there is the fervently defended claim-but really it is an unproven ideology-that all mental disorders are brain diseases in which something has gone wrong at the physiological level and has a pathogenesis describable in genetic or neurobiological terms. This leads to the default assumption that mental disorders are best treated with medication or other physiological

J. C. Wakefield ( $\square)$

Silver School of Social Work, New York University,

New York, NY, USA

e-mail: jerome.wakefield@nyu.edu interventions. Third, as DSM-5 manifests in the extreme, there is a continued dramatic expansion of the domain of disorder both through new categories and loosening of diagnostic criteria for current categories. This diagnostic expansiveness casts increasing doubt on the previous two claims about mental disorder, yet this tension is ignored. The net effect is to swallow more and more of social work's traditional domain within the medical/brain-disease model. The DSM-5, one might argue, gives the phrase "psychiatric social work" a new meaning, for psychiatry itself appears to be taking over many social work tasks, albeit under the banner of treating mental disorder (and perhaps with the intent of treating the "brain-in-environment" rather than person-in-environment).

There is a difference between mental disorder and normal-range problems of living. Mental disorders are not merely problematic or harmful; they are "harmful dysfunctions" (Wakefield 1992, 2006), in which the harm can be any negative or undesirable condition according to social values, and the dysfunction that causes the harm is the failure of some internal psychological process or mechanism to function as it was biologically designed to function. There is impressive agreement by clinicians and lay people on many judgments of which problems represent disorders and which are part of normal distress and problems of living (Wakefield et al. 2002, 2006), suggesting that the concept of disorder is widely shared even if inherently fuzzy. However, nothing in the concept of disorder determines that every psychological dysfunctionthat is, every failure of mental processes to operate as they were biologically designed to do-must be a brain dysfunction rather than a problem strictly at the level of the interaction of thoughts, emotions, and other meanings.

The primary argument put forward for the brain-disease model of mental disorder is that psychological disturbances 
must be brain diseases because psychological processes take place in the brain. This argument, advanced by leaders in the field including Nobel Prize winners (Weir 2012), is manifestly unsound and without conceptual foundation. Psychological processes in the brain can go astray at the psychological level without the brain itself having anything wrong with it (Wakefield 1997). If somebody sent to help you with a computer software glitch claimed that every software problem on your computer must be a hardware problem-after all, all software runs in hardware-so replacing the hardware is called for, you would laugh and assume the person is trying to sell you some hardware, because of course there can be software problems that are not hardware problems. The argument that every psychological disorder must be a brain disease is equally misguided, yet taken seriously. Some new schemes for conceptualizing mental disorders as brain disorders do take psychosocial factors into account as part of the overall mix of causal factors (Insel et al. 2010; White et al. 2012), but the shift to a brain-disease approach is at their core. It is often argued that classifying psychological disorders as brain diseases, even if inaccurate, is justified on humanitarian grounds because it reduces stigma, but recent studies demonstrate that when mental problems come to be seen as brain diseases, stigma sometimes increases or remains the same (Angermeyer and Matschinger 2005; Schnittker 2008; Schomerus et al. 2012). Even the common assertion that having a brain malfunction relieves the patient of the accusation of having a character defect is actually faulty, given the "brain disease" model's own assumptions; character weaknesses must also occur in the brain and are brain processes, so a brain defect may either itself be or cause a character defect. The brain disease model does none of good things it is advertised to do in return for the mischief it causes.

The combination of an invalidly expansive approach to labeling human suffering as mental disorder with an ideological fixed idea that mental disorder is brain disease has the consequence of overuse of medication for problems of living. Thus, Center for Disease Control and Prevention data show that, for example, $11 \%$ of all Americans-and a remarkable $23 \%$ of all women in their 40 and $50 \mathrm{~s}$-are taking antidepressants, and about $11 \%$ of American children - and a remarkable 1 in 5 boys in high school-are diagnosed with ADHD and likely to get stimulant medication.

Depression provides an apt example of the distorted treatment profile that derives from the brain disease model. Despite the strong evidence from outcome studies that psychotherapy works as well or better than medication for treatment of the vast majority of depression cases, and the special appropriateness of social workers for treating most cases of depression because they generally are mild or moderate cases involving social and interpersonal triggers and maintaining causes, the impact of "brain disease" ideology has been powerful. For example, analysis of Medical Expenditure Panel Survey data for 2007 (Marcus and Olfson 2010) revealed that in 2007, social workers treated only about $7 \%$ of the depression cases in the U.S., whereas physicians treated about $85 \%$ (with somewhat more non-psychiatrist physicians than psychiatrists involved); psychologists treated about $20 \%$ (percentages add up to more than $100 \%$ because some patients saw more than one professional). Moreover, the percentage of depression patients getting any psychotherapy had decreased over the previous decade from about $53 \%$ to about $43 \%$, with about $82 \%$ getting medication; of those receiving psychotherapy, about three-quarters also got antidepressants. So, about one in ten depression patients received psychotherapy as their sole treatment. This lopsided approach is not at all warranted by the existing empirical evidence.

For social workers, even from a purely practical perspective (that is, ethical issues of misdiagnosis aside; see below for those), this situation presents a dilemma. If more conditions are diagnosable, that allows more individuals to be helped. However, those individuals are likely to be targeted for drug development and end up being helped by general medical practitioners. The above example of depression is illustrative. The flawed DSM diagnostic criteria for depression criteria create an inflated number of potential diagnoses (Horwitz and Wakefield 2007), so that over half of the population likely qualifies at some point in life (Eaton et al. 1997; Moffitt et al. 2010). Moreover, as noted, this is an ideal condition to be treated by social workers. Yet, by far most depression is treated by physicians. Similar considerations apply to many other conditions, such as anxiety disorders (Horwitz and Wakefield 2012).

\section{Psychological Justice as a Goal of Clinical Social Work}

We need to start proactively moving past the limitations of disorder and to more fully conceptualize the nondisordered conditions that we treat and the reasons why they merit reimbursement. Social norms and then reimbursers may eventually follow. The mental health professions do many desirable things other than treat mental disorder. They relieve psychological suffering, provide psychological enhancement (e.g., leadership training, resilience training), and inculcate virtues, such as courage and wisdom, that allow individuals to transcend the problematic parts of their natures (Wakefield 2010).

However, from a social perspective, there is one function of the mental health professions that is as fundamental as the treatment of mental disorder itself in terms of the 
social mission of the professions. Ever since Plato's Republic, the interface between the internal psychological nature of the individual, the individual's successful social role functioning, and the overall just and beneficial functioning of society has been central to social theory and the theory of justice. Social justice inherently involves a mindin-person-in-role-in-environment perspective. Hence, a justice-oriented profession such as social work (Wakefield 1988a, b) also simultaneously and quite consistently can be the largest mental health profession (except of course for general physicians, who in this medication-oriented era treat more mental health problems than all the dedicated mental health professions combined).

I would argue that a fundamental goal of the mental health professions-one that should be kept distinct from the mandate to treat mental disorder, but is currently confusedly mixed up with disorder-is to treat normal, nondisordered human variation that happens not to match our society's demands and needs and therefore reduces the individual's opportunity for social participation, contribution, and fulfillment. This might be called "psychological justice"-equipping individuals with a fair complement of the psychological features that our society demands. Of all the mental health professions, social work is unique in conceiving its mission as going beyond treatment of mental disorder to seeking social justice through a proper fit between individuals' psychological capacities and the social environment.

It is a cruel and shortsighted policy that our society currently refuses to be as generous in supporting help for the nondisordered as for the disordered. Surely it is in society's interest to offer help, for example, to couples with serious conflicts that might break apart a family, even if marital incompatibility need not represent a mental disorder in either partner. In the medical realm, many nondisorders are socially mandated to be treated and reimbursed because that is seen as just and necessary-from provision of birth control to relief of the normal pain of childbirthand the same should be true of some nondisordered psychological problems. Many people have normal variations that make them less able to function adequately in the novel social environments we as a society have constructed around them, and they deserve our help. If, for example, an individual feels natural social anxiety when speaking before groups of strangers and thus cannot succeed in many of today's professions in our mass society, or if the individual has a normal circadian rhythm and cannot easily adapt to shift work demanded by an available job, the individual deserves help, and deserves it without being spuriously labeled as having "social anxiety disorder" or "circadian rhythm disorder, shift work type." A natural fear of heights may keep someone from certain occupations, and someone with intense prolonged grief-not necessarily a disorder, but often related to temperament and the unique nature and meaning to the individual of the relationship that was lost-may not able to function satisfactorily in our efficiency conscious society and may lose needed resources as a result. These individuals deserve help without having spurious labels of "specific phobia" or "persistent complex grief disorder." Hopefully eventually our society will recognize that it is outrageous, unjust, and socially harmful to fail to offer help for a variety of psychological conditions that are not disorders but are generated by the interaction of normal human nature with the novel social environment that we are creating, and will allow us to diagnose and obtain reimbursement accordingly, just as it does now for nondisordered conditions related to reproductive health.

\section{Introducing the DSM-5}

This issue of CSWJ begins with a DSM-5 primer (Wakefield 2013a) that selectively surveys the major DSM-5 changes and the controversies that broke out over them, with special attention to the depressive disorders. The overall picture is of a relatively consistent expansion of the Manual's domain of disorders through both broad new categories and expansions to the diagnostic criteria of existing categories. It is this trend towards medicalization that was the primary target of the DSM's critics, and remains a point of contention. This trend was especially of concern due to the likelihood, as noted, that once conditions are classified as disorders they would become the target of drug development efforts. Indeed, the rationale for several additions to the Manual was that medication exists to improve the condition-although this argument for something being a disorder is manifestly fallacious, and the evidence for medication effectiveness was in some cases laughably weak (Wakefield 2013b). The net result of all this controversy is that psychiatry itself is divided about whether the DSM-5 provides an improved guide to diagnosing mental disorder, and critics from within psychiatry are advising clinicians to use their own judgment (Frances 2012).

\section{The "Alternative" Personality Disorders Diagnosis System in DSM-5}

The next three papers in this issue introduce the reader to the proposed new trait-based dimensional system for diagnosing personality disorders (PDs), and to the controversies over it. The new system is first outlined with admirable lucidity by Lanier et al. (2013). One of the coauthors of this paper, Robert Krueger, a psychologist, 
has been heavily involved in the research that provided the foundation for the new approach. The alternative PD system is supposed to resolve the sorts of issues that concerned the DSM-5 architects, such as too much use of "NOS" categories and too much comorbidity. The system is based on the idea is that each PD essentially consists of extreme personality traits, and thus the best way to diagnose PD is to rate the individual patient on a comprehensive set of personality traits and use those ratings as the diagnosis.

A caveat is critical here. The new PD system was supposed to be the centerpiece in DSM-5's attempt to introduce dimensional diagnosis into the Manual. At the time these papers were accepted, it appeared that this proposed new system would entirely or partially replace the current PD categories. However, despite several comprehensive revisions, continued vigorous objections from personality disorder experts due to the complexity and untried nature of the proposed system caused the proposal to be sidelined. The familiar DSM-IV diagnostic categories and criteria were retained in the main DSM-5 listing and will be available for the clinician's use, at least until the planned interim partial online revision (DSM-5.1) within the next few years. The proposed dimensional system was instead placed in DSM-5's section 3, for proposals still being studied. It is being portrayed as a parallel "alternative system" of PD diagnosis that can be used immediately under the "otherwise specified" category in the main listing. Further research on the new system is proceeding, and it is expected that this system, or something very similar, will formally replace the current categories. Thus, grappling with this "alternative" system is warranted.

The PD proposal also illustrates the crosscurrents of controversy in the DSM-5 process. Tom Widiger, also a psychologist who is a leading personality disorders researcher, offers a trenchant critique of the popular "prototype matching" approach to PD diagnosis that was considered at one point, and comments critically on the final alternative system (he notes as well that the final system is not precisely the one Lanier et al. describe). He notes that an eminent member of the PD work group, John Livesley, withdrew and penned a scathing critique of the proposal (Livesley 2010). Personality psychologists have developed a well-known and much-researched "five factor" dimensional systems of trait ratings to characterize normal personality. Because this standard system of trait analysis did not seem to adequately capture some forms of pathology, the alternative PD system was constructed somewhat differently, to include more pathology-related dimensions and traits. The jerry-rigged nature of this system is critiqued by Widiger (as it was by Livesley), who prefers the more research-based five-factor structure. Note that all these researchers prefer a trait-based dimensional system, but disagree about its precise nature based on their own research programs. One might worry that this is a case of too many researchers spoiling the clinical broth.

I have argued elsewhere that in fact personality is the overall interaction among traits and that personality disorders cannot and should not be atomized into sets of traits (2008). One concern is that, unlike categories with their symptom thresholds, the trait approach to PD, being dimensional, does not come with any automatic thresholds for diagnosis built into it. Thus, in effect, a personality disorder could be any configuration of personality traits that had high ratings on some negative traits. Such a category could potentially include almost everyone (Wakefield 2008). To try to address this worry, the alternative PD system precedes the trait analysis by an evaluation of whether the person satisfies the general definition of $\mathrm{PD}$, which is strengthened from its weak DSM-IV version. In my commentary on the alternative PD system, I detail the history and structure of the new general definition of personality disorder (Wakefield 2013c). I argue that, while the new definition is an improvement on DSM-IV's definition, it is still too weak to prevent a potential explosion of questionable PD diagnosis.

\section{DSM-5 Dilemmas for Social Work Clinicians and Educators}

Probst's (2013) paper provides a rare and nuanced qualitative empirical study of how social workers actually use the DSM. She specifically explores how social workers negotiate the tension between the mental-disorder perspective of the DSM with the person-in-environment perspective of the social work profession. Her account of her informants' views-with which I believe most readers will empathize-reveals their acute awareness of the distinction between mental disorder and problems of living, their realization that the DSM's diagnostic criteria for disorder often do not draw the disorder/normal distinction adequately, and their sensitivities to the ethical dilemmas resulting from the complex mismatches between DSM criteria, true mental disorder, and client needs for help whether disordered or normally distressed.

Once the clinician diagnoses disorder and locates the primary source of the problem within the individual, this tends to constrain treatment choices (Garb 1999). Whether someone has a mental disorder or not, matching the individual to a social niche that fits their needs and capacities should be an integral part of any intervention strategy. To this extent, clinical intervention into the person-environment interaction is "blind" regarding the disorder/normal distinction. As Probst mentions, I (Wakefield 1996a, b) have argued that attention to the environment is not a matter of applying some general "ecosystems" framework, 
because such a framework provides no real guidance for the clinician. Some problems need individual change, some environmental change, and many some mixture of both. For each problem domain, domain-specific causal theories are needed to guide understanding of that particular domain. No client deserves a cookie cutter case formulation-whether the cookie cutter be "brain disease" or "ecosystems" ideology.

Probst documents how social workers face challenging dilemmas in using the DSM. A common and appealing solution is to play the DSM game to obtain reimbursement, but keep your head about what is really going on with your client. This requires recording diagnoses that may not be what the clinician really thinks. The ethical and legal implications did not escape Probst's informants, some of whom stated bluntly, "It is fraud." The occurrence of such intentional misdiagnosis is very widespread. Kirk and Kutchins's (1988) study of social work misdiagnoses is often cited, but this is not just a social work issue. In one study (Rost et al. 1994), almost half of all physicians said they purposely misdiagnosed depression in order to get higher reimbursements that were paid for more physical diagnoses. The physicians, like the social workers in Probst's study, recognized that deliberate misdiagnosis may constitute fraud (Hsia and Tam 1991; Jesilow et al. 1991; Morreim 1988), yet, like social workers, saw it as morally acceptable because it was the lesser of two evils. My informal questioning of colleagues has yielded similar "findings"; a psychiatrist and psychologist I recently questioned both said that of their depressed and ADHD patients, respectively, about $75-80 \%$ are probably normal individuals having difficulty adapting to circumstances, whereas maybe $20-25 \%$ genuinely have something internally wrong with their mental functioning. We obviously need distinctions, not one-size-fits-all ideology.

The diagnostic situation is perplexing, to say the least. Is it fraud to classify a condition as a reimbursable mental disorder if it does actually satisfy the diagnostic criteria of a DSM category of disorder, yet the category itself is defined in an overly expansive style that, the clinician truly believes, mistakenly classifies problems in living as mental disorders? Can two wrongs make a right when it comes to helping a patient?

The DSM uses descriptive rather than etiology-based categories in which disorders are defined mostly at the symptom level rather than in terms of deeper processes including unconscious processes. Damon Krohn (2013) argues that the DSM's influence on education, along with rise of cognitive-behavioral therapy and the widespread educational use of DSM-category-based treatment manuals originally developed for research, has eclipsed valuable aspects of psychoanalytic thinking in social work education. The result is lack of student sensitivity to the importance of empathy, affect, and unconscious processes. Krohn explores the resulting changes that, he argues, are one-dimensionalizing clinical education and practice.

Kohut's emphasis on empathy-in both verbal and nonverbal forms-as a kind of direct observation of the patient's internal world fits well with the recent discovery of mirror neurons, revealing that we are biologically designed to model the internal workings of those around us and to some degree experience in experimental form what they are experiencing (Eagle and Wakefield 2007). That said, one must remain wary of the inflated faith among some analysts in countertransference as a conduit to the truth about the patient. Krohn usefully reminds us that "It is the commitment to steadfast exploration into the client's subjectivity and not sympathy that strengthens the therapeutic alliance."

One diagnostic example of Krohn's concern about trading affect for cognition due to the rise of the cognitivebehavioral model has occurred in the DSM itself. Generalized anxiety disorder (GAD) originally referred to Freud's notion of undirected, free-floating anxious affect that is not consciously directed at any particular feared mental content, a clear pathology. However, cognitive theorists on the anxiety disorders work group during a DSM revision noticed that this classic conception does not fit their theory; for them, all affect must be immediately aroused by a cognition, by which they generally mean conscious cognition. Consequently, GAD was transformed into a "worry disorder" (there was actually a move to so rename it this time around) in which one has several "excessive" worries, which are cognitively based directed fears or anxieties about specific perceived threats. This ignores the reality of undirected affect that this category was created to capture, which (short of panic disorder) is no longer diagnosable under any specific DSM category (except "NOS"). The change also further blurs the boundaries between disorder and normality, for even worries that are objectively "excessive" about children, health, finances, or terrorism can be part of normal overall functioning in a way that chronic free-floating anxious arousal is not.

Our grappling with diagnostic dilemmas in this issue of CSWJ closes with two invited commentaries. The first is by Phillips (2013) on the ethical and legal dimensions of misdiagnosis. Phillips picks up Probst's theme of the dilemma of the social work clinician: "more and more clinical social workers have earned the legal authority to diagnose individuals who suffer from various mental disorders and, at the same time, are required to do so using a system which many feel to be incompatible with the principles and values of the social work profession." Probst quotes me as having expressed an accepting attitude towards misdiagnosing normal distress as mental disorder 
for reimbursement purposes. However, after reading Phillips's discussion, I am not so sure. His lucid and illuminating - and sometimes anxiety-provoking-discussion of the legal issues involved in misdiagnosis suggests that in the long run it is not a viable option, or at least it is a dangerous option, for the clinician to "play the game" of DSM diagnosis knowingly offering what in fact the clinician considers misdiagnoses. Phillips's analysis is aimed primarily at underdiagnosis to prevent stigma or overdiagnosis to obtain reimbursement. In fact, with the DSM-5 controversies, the situation is even more complex. In DSM5 , many conditions classified as mental disorders are likely not genuine disorders. Thus, even by faithfully following the rules (i.e., the diagnostic criteria), one is, in another sense, not following the rules (i.e., one is classifying as mental disorders conditions that one does not believe are disorders). An accurate diagnosis by DSM standards may be a deception by the clinician's own standards. As noted, Frances (2012), the Editor of DSM-IV and the a vehement and persistent critic of DSM-5's overreaching, advises clinicians to simply ignore the DSM-5's criteria and not to overpathologize one's patients. The legal and moral dimensions of such a situation have yet to be explored, but Phillips's commentary provides a good place to start in thinking about the ramifications of one's choices.

Toto-Moriarty and Mastria (2013), in their invited commentary, argue that the expansion of the DSM-5's eating disorders to include the new category of binge eating disorder-which will now immediately become the most common eating disorder, due to its high prevalence in the population-opens up the possibility for expanded clinical intervention, including psychodynamic intervention, with people in need of clinical services. They distinguish those with focal eating problems from those with broader psychological determinants of disordered eating, an essential distinction that is lost within the DSM categorical diagnosis but must be brought into case formulation. (This point resonates with my memory of that time long ago when therapists were enthralled with Masters and Johnson sex therapy and its remarkable cure rates, until after a few years they realized that the "easy" more focal sex-difficulty cases were becoming rarer and those with deeper issues behind the sex problem were emerging to yield much lower success rates.) Toto-Moriarty and Mastria's article illustrates how a theoretically integrative, individualized treatment perspective with multiple intervention strategies at one's command is crucial to meeting the needs of the diverse populations and etiologies that are covered by even a single DSM category. Toto-Moriarty and Mastria thus help us to see how the clinician can evade the cookiecutter mentality that Krohn suggests is creeping into our field's educational practices as a result of manualized DSM-category-based treatment guides.
Frances (2012) objects that the BED category will simply pathologize normal gluttony. From an evolutionary perspective on normality, one might argue that, having been evolutionarily shaped at a time when food calories were difficult to come by, human beings naturally tend to have a taste preference for high-calorie sweets and fat and are inclined to overeat when good-tasting food is available, for in natural environments food might not be available later on. Like all human traits, individuals' inclination to be opportunistic eaters, and their internal balance between eating tendencies and inhibitory control abilities, vary enormously in ways that were fine in the environments for which we became biologically adapted. So, one might ask, is it really a mental disorder to overeat once a week for 3 months and (being subject to our society's judgmental attitudes about weight and exaggerated notions of rational control) then to feel out of control and disgusted with oneself as a result? Or, is such behavior simply an imbalance between a normal variant of the way human nature is biologically designed and the novel environment we have created of readily available plentiful high calorie goodtasting food all around us? Whichever it is-mental disorder or socially disvalued consequence of normal human variation interacting with the social environment-people who have this problem deserve help. One might be concerned that the new diagnostic category encourages facile labeling of enormous numbers of individuals, rather than individual understanding and acceptance of diversity, and it also opens the way for drug development to control individuals rather than strategies that address the problematic individual/environment interaction that is at the root of the problem. Toto-Moriarty and Mastria's nuanced and thoughtful comment encourages the hope that, despite such pitfalls, clinicians can use the new diagnosis wisely and fruitfully.

We will clearly be living with both the challenges and opportunities presented by the DSM- 5 for some time. Allow me, however, to close with a broadly optimistic speculation about the future. The ICD-10 comes online in 2014, and then not too far in the future the new ICD-11, which is now in process of being created, will also be published and eventually adopted in the U.S. The DSM by law must coordinate with the ICD and it must use the same codes (the DSM-5 is already prepared for the switch to ICD-10 with duplicate coding). The WHO is much more cognizant of cross-cultural issues and sensitive to social context than is the APA, and it also reflects the Europeans' lesser concern with disorder per se as a requirement for reimbursement of mental health services. The pressure to conform with the ICD is responsible, for example, for the DSM-5's greatly expanded (but still selective, relative to the ICD) listing of "V Code" conditions that are not disorders but often treated, which might be seen as a growing 
approximation to a social work person-in-environment diagnostic system that is not pathology based existing within the DSM itself. Out of the turmoil and disappointment over DSM-5's limitations and the ongoing interaction of the DSM with the ICD, supplemented by our profession's insistence that psychological justice is a legitimate socially supported task of the mental health professions along with treatment of mental disorder, there may lie the opportunity for our society's embrace of a more humane and conceptually sensible diagnostic and reimbursement system in the years to come. One can hope that such developments would allow a resolution of some of the conflicts that social workers now experience in using the DSM.

\section{References}

Angermeyer, M. C., \& Matschinger, H. (2005). Causal beliefs and attitudes to people with schizophrenia: Trend analysis based on data from two population surveys in Germany. British Journal of Psychiatry, 186, 331-334.

Eagle, M. N., \& Wakefield, J. C. (2007). Gestalt psychology and the mirror neuron discovery. Gestalt Theory-An International Multdisciplinary Journal, 29, 59-64.

Eaton, W. W., Anthony, J. C., Gallo, J., Cai, G., Tien, A., Romanosky, A., et al. (1997). Natural history of diagnostic interview schedule/DSM-IV major depression: The baltimore epidemiologic catchment area follow-up. Archives of General Psychiatry, 54, 993-999.

Frances, A. (2012). DSM-5 is a guide, not a bible: Simply ignore its 10 worst changes. http://www.huffingtonpost.com/allen-frances/ dsm-5_b_2227626.html.Accessed 25 Dec 2012.

Garb, H. N. (1999). Studying the clinician: Judgment research and psychological assessment. Washington, DC: American Psychological Association.

Horwitz, A. V., \& Wakefield, J. C. (2007). The loss of sadness: How psychiatry transformed normal sorrow into depressive disorder. New York: Oxford University Press.

Horwitz, A. V., \& Wakefield, J. C. (2012). All we have to fear: Psychiatry's transformation of natural anxieties into mental disorders. New York: Oxford University Press.

Hsia, D. C., \& Tam, Q. (1991). Suing physicians who make false claims. Annals of Internal Medicine, 114, 1050-1053.

Insel, T., Cuthbert, B., Garvey, M., Heinssen, R., Pine, D. S., Quinn, K., et al. (2010). Research Domain Criteria (RDoC): Toward a new classification framework for research on mental disorders. American Journal of Psychiatry, 167, 748-751.

Jesilow, P., Geis, G., \& Pontell, H. (1991). Fraud by physicians against Medicaid. JAMA, 266, 3318-3337.

Kirk, S. A., \& Kutchins, H. (1988). Deliberate misdiagnosis in mental health practice. Social Service Review, 62(2), 225-237.

Krohn, D. (2013). The three fallacies: Evaluating three problematic trends in clinical practice. Clinical Social Work Journal (this issue).

Lanier, P., Bollinger, S., \& Krueger, R. (2013). Advances in the conceptualization of personality disorders: Issues affecting social work practice and research. Clinical Social Work Journal (this issue).

Livesley, W. J. (2010). Confusion and incoherence in the classification of personality disorder: Commentary on the preliminary proposals for DSM-5. Psychological Injury and Law, 3, 304-313.

Marcus, S. C., \& Olfson, M. (2010). National trends in the treatment for depression from 1998 to 2007. Archives of General Psychiatry, 67(12), 1265-1273.

Moffitt, T. E., Caspi, A., Taylor, A., Kokaua, J., Milne, B. J., Polanczyk, G., et al. (2010). How common are common mental disorders? Evidence that lifetime prevalence rates are doubled by prospective versus retrospective ascertainment. Psychological Medicine, 40, 899-909.

Morreim, E. H. (1988). Cost containment: Challenging fidelity and justice. Hastings Center Report, 18, 20-25.

Phillips, D. (2013). Clinical social workers as diagnosticians. Clinical Social Work Journal (this issue).

Probst, B. (2013). "Walking the tightrope:" Clinical social workers' use of diagnostic and environmental perspectives. Clinical Social Work Journal (this issue).

Rost, K., Smith, G. R., Matthews, D. B., \& Guise, B. (1994). The deliberate misdiagnosis of major depression in primary care. Archives of Family Medicine, 3, 333-337.

Schnittker, J. (2008). An uncertain revolution: Why the rise of a genetic model of mental illness has not increased tolerance. Social Science \& Medicine, 67, 1370-1381.

Schomerus, G., Schwahn, C., Holzinger, A., Corrigan, P. W., Grabe, H. J., Carta, M. G., et al. (2012). Evolution of public attitudes about mental illness: A systematic review and meta-analysis. Acta Psychiatrica Scandinavica, 125, 440-452.

Toto-Moriarty, T, \& Mastria, M. R. (2013). Eating Disorder Changes in the DSM-V: Clinical Implications. Clinical Social Work Journal (this issue).

Wakefield, J. C. (1988a). Psychotherapy, distributive justice, and social work: I. Distributive justice as a conceptual framework for social work. Social Service Review, 62, 187-210.

Wakefield, J. C. (1988b). Psychotherapy, distributive justice, and social work: II. Psychotherapy and the pursuit of justice. Social Service Review, 62, 353-382.

Wakefield, J. C. (1992). The concept of mental disorder: On the boundary between biological facts and social values. American Psychologist, 47, 373-388.

Wakefield, J. C. (1996a). Does social work need the eco-systems perspective? Part 2. Does the perspective save social work from incoherence? Social Service Review, 70, 183-213.

Wakefield, J. C. (1996b). Does social work need the eco-systems perspective? Part 1 . Is the perspective clinically useful? Social Service Review, 70, 1-32.

Wakefield, J. C. (1997). Diagnosing DSM-IV, Part 1: DSM-IV and the concept of mental disorder. Behavior Research and Therapy, $35,633-650$.

Wakefield, J. C. (2006). The concept of mental disorder: Diagnostic implications of the harmful dysfunction analysis. World Psychiatry, 6, 149-156.

Wakefield, J. C. (2008). The perils of dimensionalization: Distinguishing personality traits from personality disorders. Psychiatric Clinics of North America, 31, 379-393.

Wakefield, J. C. (2010). False positives in psychiatric diagnosis: Implications for human freedom. Theoretical Medicine and Bioethics, 31(1), 5-17.

Wakefield, J. C. (2013a). DSM-5: An overview of changes and controversies. Clinical Social Work Journal (this issue).

Wakefield, J. C. (2013b). The DSM-5 debate over the bereavement exclusion: Psychiatric diagnosis and the future of empirically supported practice. Clinical Psychology Review. Published online: http://dx.doi.org/10.1016/j.cpr.2013.03.007.

Wakefield, J. C., (2013c). DSM-5 and the general definition of personality disorder. Clinical Social Work Journal (this issue). 
Wakefield, J. C., Kirk, S. A., Pottick, K. J., Tian, X., \& Hsieh, D. K. (2006). The lay concept of conduct disorder: Do non-professionals use syndromal symptoms or internal dysfunction to distinguish disorder from delinquency? Canadian Journal of Psychiatry, 51, 210-217.

Wakefield, J. C., Pottick, K. J., \& Kirk, S. A. (2002). Should the DSM-IV diagnostic criteria for conduct disorder consider social context? American Journal of Psychiatry, 159, 380-386.

Weir, K. (2012). The roots of mental illness: How much of mental illness can the biology of the brain explain? APA Monitor, 43(6), 30.

White, P. D., Rickards, H., \& Zeman, A. Z. J. (2012). Time to end the distinction between mental and neurological illnesses. BMJ, 344, e3454.

Widiger, T. (2013). Changes in the conceptualization of personality disorder: The DSM-5 debacle. Clinical Social Work Journal (this issue).

\section{Author Biography}

Jerome C. Wakefield PhD, DSW, MSW, LCSW is University Professor, Professor of Social Work, and Professor of Psychiatry at New York University. He is the coauthor (with Allan Horwitz) of "The Loss of Sadness: How Psychiatry Transformed Normal Sorrow into Depressive Disorder" (Oxford 2007), named best psychology book of 2007 by the Association of Professional and Scholarly Publishers, and "All We Have to Fear: How Psychiatry Transforms Natural Fear into Mental Disorder" (Oxford 2012). He can be contacted at jerome.wakefield@nyu.edu. 